\title{
A search for the in trans role of GraL, an Escherichia coli small RNA*
}

\author{
Maciej Dylewski, Monika Ćwiklińska\# and Katarzyna Potrykus ${ }^{\varpi}$ \\ Department of Bacterial Molecular Genetics, Faculty of Biology, University of Gdansk, Gdańsk, Poland
}

\begin{abstract}
Small RNA are very important post-transcriptional regulators in both, bacteria and eukaryotes. One of such sRNA is GraL, encoded in the greA leader region and conserved among enteric bacteria. Here, we conducted a bioinformatics search for GraL's targets in trans and validated our findings in vivo by constructing fusions of probable targets with IacZ and measuring their activity when GraL was overexpressed. Only one target's activity (nudE) decreased under those conditions and was thus selected for further analysis. In the absence of GraL and greA, the nudE::lacZ fusion's $\beta$-galactosidase activity was increased. However, a similar effect was also visible in the strain deleted only for greA. Furthermore, overproduction of GreA alone increased the nudE::IacZ fusion's activity as well. This suggests existence of complex regulatory loop-like interactions between GreA, GraL and nudE mRNA. To further dissect this relationship, we performed in vitro EMSA experiments employing GraL and nudE mRNA. However, stable GraL-nudE complexes were not detected, even though the detectable amount of unbound GraL decreased as increasing amounts of nudE mRNA were added. Interestingly, GraL is being bound by $\mathrm{Hfq}$, but nudE easily displaces it. We also conducted a search for genes that are synthetic lethal when deleted along with GraL. This revealed 40 genes that are rendered essential by GraL deletion, however, they are involved in many different cellular processes and no clear correlation was found. The obtained data suggest that GraL's mechanism of action is non-canonical, unique and requires further research.
\end{abstract}

Key words: GraL, GreA, sRNA, sRNA targets, synthetic lethal genes

Received: 01 February, 2018; revised: 26 February, 2018; accepted: 03 March, 2018; available on-line: 12 March, 2018

e-mail: katarzyna.potrykus@biol.ug.edu.pl

"Present address: Institute of Physical Chemistry of the Polish Academy of Sciences, Kasprzaka 44/52, 01-224 Warsaw, Poland

*A preliminary report on this subject has been presented at the Molecular Genetics of Bacteria and Phages Meeting, Madison, Wisconsin, USA (2016)

Abbreviations: EMSA, electrophoretic mobility shift assay; IPTG, isopropyl $\beta$-D-1-thiogalactopyranoside; ppGpp, guanosine-3',5'-bisdiphosphate; $p p G p p^{0}$ strain, strain devoid of ppGpp; sRNA, small RNA; X-gal, 5-bromo-4-chloro-3-indolyl $\beta$-D-galactopyranoside

\section{INTRODUCTION}

Regulatory RNAs that control gene expression posttranscriptionally are commonly found in prokaryotic and eukaryotic worlds. In eukaryotes, they are called noncoding RNAs and are represented by miRNA and siRNA. In prokaryotes, such RNAs are referred to as small RNA (sRNA) (Gottesman, 2004; Gottesman \& Storz,
2011). In recent years a growing number of sRNA has been reported, reaching over 900; this includes about 90 sRNA in Escherichia coli alone (according to sRNAmap, a small RNA database (Huang et al., 2009)). It is evident that sRNA are crucial for bacterial regulation of many processes, for example by controlling expression of major transcriptional factors (like RpoS in E. coli (Gottesman, 2004; McCullen et al., 2010)), outer membrane protein genes (Guillier \& Gottesman, 2006; Valentin-Hansen et al., 2007), quorum sensing (Tu \& Bassler, 2007), iron homeostasis (Masse et al., 2005; Masse et al., 2007; Vecerek et al., 2007), LPS modification (Moon \& Gottesman, 2009) and pathogenicity (Romby et al., 2006; Toledo-Arana et al., 2007).

sRNA are 50-500 nucleotides long and may act either in cis or in trans (Storz et al., 2011). Cis-acting sRNA exert their effects directly on the gene in the locus that encodes them and include riboswitches and anti-sense RNAs. Riboswitches are embedded in the mRNA structure whose expression they regulate; upon binding of an effector molecule or responding to an environmental signal, secondary structure of the riboswitch changes and often either allows or blocks transcription or translation (Gottesman \& Storz, 2011; Kortmann \& Narberhaus, 2012; Hoe et al., 2013). Anti-sense RNAs require perfect base pairing with their targets (Pedersen \& Gerdes, 1999; Kawano et al., 2002).

On the other hand, trans-acting sRNA exert their effects on genes localized in a different locus than the one that encodes them. Such regulation is usually dependent on imperfect base pairing between a given sRNA and its target mRNA(s) (Gottesman, 2004; Storz et al., 2011). One sRNA may regulate many genes, for example DsrA targets $\operatorname{rcs} A$, bns, $r p o S$, ilvH and $\operatorname{argR}$ mRNA (Sledjeski \& Gottesman, 1995; Lease et al., 1998; Majdalani et al., 2005). The mechanism of action may exert a positive effect on gene expression and involve uncovering of the ribosomal binding site; or negative, where ribosomal binding site might be blocked or the mRNA might be targeted for degradation (Gottesman \& Storz, 2011). Often, but not always, base pairing requires an additional factor, i.e. Hfq protein that is thought to stabilize the RNA-RNA interactions (Beisel et al., 2012; Faner \& Feig, 2013).

The majority of sRNA are trans-acting. Still, only a fraction of those sRNA have known mRNA targets (according to sRNAmap database only about half of E. coli sRNA have known targets). GraL, a small regulatory RNA that we discovered while investigating gre $A$ regulation (Potrykus et al., 2010), is an example of sRNA whose targets still remain elusive. GreA is a protein transcriptional factor that binds directly to RNA polymerase and affects the initiation and elongation steps of transcription 
(Laptenko et al., 2003; Borukhov et al., 2005; Potrykus et al., 2006). Thus, gre $A$ regulation is of substantial general interest, especially in the light of a discovery that there is a tight interplay between three transcriptional factors of similar structure - GreA, GreB, and DksA (Potrykus et al., 2006; Aberg et al, 2008; Vinella et al., 2012).

In our previous work (Potrykus et al., 2010), we found that gre $A$ promoter region contains two very strong overlapping promoters ( $\sigma^{70}$ dependent P1 promoter, and $\sigma^{\mathrm{E}}$ dependent P2 promoter, whose transcription start sites are $10 \mathrm{bp}$ apart) and an unusual terminator, localized in this gene's $149 \mathrm{nt}$ leader region (Fig. S1 at www. actabp.pl). The terminator causes premature transcription termination of about $2 / 3$ of the overall transcripts initiating from the P1 and P2 gre $A$ promoters (only $1 / 3$ of transcripts reads through the terminator and yields gre $A$ mRNA). Such transcripts were observed to appear both, under in vitro and in vivo conditions, and the short transcripts were termed GraL (for gre $A$ leader region) (Potrykus et al. 2010).

The effect of overproducing GraL was assessed by fitness assays and transcriptional microarrays (Potrykus et al., 2010). In mixed populations, GraL-overexpressing cells were found to overtake wild type cells subjected to several cycles of growth in liquid medium until stationary phase and then diluting back into a fresh medium, as compared to cells where a scrambled version of the GraL sequence was being overproduced. This indicated that the GraL effect is sequence specific. However, it is unclear at which phase of growth GraL conferred this fitness advantage. On the other hand, microarray data revealed that GraL significantly affects expression of over 100 genes, which includes genes for motility, iron homeostasis and amino acid metabolism. However, bioinformatics search for sequence complementarity among the affected mRNAs did not point to any one of them as being the direct target of this sRNA, and so the role and precise function of GraL still remained unsolved. A bioinformatics search with the program TargetRNA, the first program specifically designed to elucidate sRNA targets (Tjaden et al., 2006), gave no satisfactory results as well.

Here, we took advantage of a more recently released software called sTarPicker that is thought to largely outperform TargetRNA (Ying et al., 2011; Faner \& Feig, 2013). With the help of this program we were able to predict 13 highly probable targets of GraL. LacZ fusions were constructed for each of the predicted target sites and the effect of GraL on their $\beta$-galactosidase activity was measured in vivo. Among them, one predicted target (nudE) gave the most promising results and was further tested in vitro. We have also undertaken a search for genes that are synthetic lethal with GraL, in hopes of defining a biological role for this sRNA. The results obtained point to 40 genes in the absence of which GraL seems to become essential for the E. coli cells.

\section{MATERIALS AND METHODS}

Bacterial strains and plasmids. The backgrounds, genotypes, and sources of the E. coli strains used in this study are listed in Table S1 (at www.actabp.pl) in the supplemental material. All strains used are derivatives of MG1655. Mutant alleles were introduced into this background via standard P1 transduction (Miller, 1972), selecting transductants with the appropriate antibiotic resistance.

To construct predicted GraL target region translational fusions with lacZ, PCR amplifications of chromosomal
DNA from the MG1655 strain were carried out using the primer pairs listed in Table S2 (at www.actabp.pl). The fusions contained native promoter regions of each putative target gene. The PCR products were digested by EcoRI/BamHI and cloned into pRS414 (Simons et al., 1987). When single-copy fusions were employed, the fusions were introduced into the bacterial chromosome of strains CF15617 (MG1655 AlacZ) and CF15615 (MG1655 $\Delta$ lacZ $\Delta$ relA $\Delta s p o T$ ) using the RS45 lambda phage; we verified that the strains were monolysogens as described in (Powel et al., 1994). The single-copy fusions were also confirmed by sequencing to contain the proper promoter region.

The $\Delta g r e A:: \mathrm{cm}$ and $\Delta g r e A \Delta \mathrm{GraL}:: \mathrm{cm}$ strains were constructed by employing lambda Red recombination system, supplied on the pSim19 plasmid (Sharan et al., 2009). Plasmid pLysE (Studier, 1991) served as a source of the chloramphenicol resistance cassette, which was amplified with the primers listed in Table S2 (at www. actabp.pl).

Plasmids pGraL and pScr (expressing corresponding GraL or Scr RNA) were constructed by using dsDNA fragments obtained by mixing appropriate, partially overlapping primer pairs (final concentration of each primer was $1 \mu \mathrm{M}$ ), with a PCR master mix containing Taq polymerase, dNTPs and buffer (GoTaq $2 \times$ Master Mix Green, Promega; reaction final volume was $200 \mu \mathrm{l})$ followed by denaturation $\left(95^{\circ} \mathrm{C}, 1 \mathrm{~min}\right)$, cooling at $0.1^{\circ} \mathrm{C} / \mathrm{s}$ to $55^{\circ} \mathrm{C}$, incubation at $55^{\circ} \mathrm{C}$ for $1 \mathrm{~min}$, and 1 cycle of extension at $72^{\circ} \mathrm{C}$ for $1 \mathrm{~min}$. Thus prepared DNA fragments were purified with an Amicon Ultra $10 \mathrm{~K}$ device (Merck), digested by EcoRI/HindIII and cloned into the pHM1786 plasmid (Potrykus et al., 2006), under ptac promoter. Plasmid pHM1786 is a pGB2 derivative (ori pSC101, Spc ; (Churchward et al., 1984)). Primers used are listed in Table S2 (at www.actabp.pl). When needed, pHM1883 (Vinella et al., 2012) was used as a vector control. pRC-GraL was constructed by cloning the same dsDNA fragment as for pGraL, but using pRC7 as the recipient vector (de Boer et al., 1989; Bernhardt \& de Boer, 2004). Plasmid used for GreA overexpression was pHM1873 (Vinella et al., 2012), which is a pGB2 derivative.

$\beta$-galactosidase assays. $\beta$-galactosidase assays were performed as described previously (Miller et al, 1972).

Electrophoretic mobility shift assays (EMSA). These assays were performed basically as described in (Morita et al., 2012). GraL was labeled at the 5' end with Cy5 (labeled RNA oligo ordered from IDT DNA). RNA (nudE and lacZ mRNAs) was synthesized using MAXIscript ${ }^{\mathrm{TM}}$ T7 Transcription Kit (Thermo Scientific), and purified by PAGE according to the manufacturer's protocol. In an instance when labeled mRNA was employed, fluorescein-12-UTP (Roche) was used for nudE and lacZ mRNA synthesis in vitro. DNA templates for in vitro transcription were prepared by PCR (Hybrid DNA polymerase, EURx) using E. coli MG1655 suspension as source of DNA template for nudE probes, and pUC19 plasmid as a source of lac $Z$, and then purified with GenElute PCR Clean-Up Kit (Sigma/Merck). Primers used are listed in Table S2 (at www.actabp.pl).

Assays without Hfq: GraL and mRNA were mixed on ice, denatured at $70^{\circ} \mathrm{C}$ for $5 \mathrm{~min}$., incubated at $30^{\circ} \mathrm{C}$ for 1 hour and applied on a running non-denaturing $4.5 \%$ polyacrylamide gel $(0.5 \times$ TBE running buffer, $250 \mathrm{~V}$, $60 \mathrm{~min})$. GraL final concentration was $10 \mathrm{nM}$, and the nudE and lac $Z$ mRNA concentrations varied accordingly (GraL:mRNA ratios in most cases were 1:2; mRNA ti- 
tration experiment was done with $1: 2,1: 4,1: 8$ or $1: 16$ ratios).

Assays with Hfq: GraL was mixed with $\mathrm{Hfq}$ and mRNA on ice, incubated for $20-30 \mathrm{~min}$. at $37^{\circ} \mathrm{C}$ and applied on a running non-denaturing $6 \%$ polyacrylamide, $5 \%$ glycerol gel $(0.5 \times$ TBE running buffer, $250 \mathrm{~V}, 90$ $\mathrm{min})$. Final concentrations were: $\mathrm{GraL}-7.5 \mathrm{nM}$, Hfq $300 \mathrm{nM}$ (monomers, corresponding to $50 \mathrm{nM}$ Hfq hexamers). The nudE and lac $Z$ mRNA concentrations varied accordingly (GraL:mRNA ratios in most cases were 1:2; mRNA titration experiment was done with 1:2, 1:4, 1:8 or 1:16 ratios). Purified native $\mathrm{Hfq}$ was a generous gift received from Dr. Grzegorz M. Cech.

Construction of a library of strains that are synthetic lethal with GraL. The library of synthetic lethal strains was constructed basically as described in (Bernhardt \& de Boer, 2004). Strain ECMZ1506 (ppGpp ${ }^{0}$ $\triangle$ greA $\triangle \mathrm{GraL} / \mathrm{pRC}-\mathrm{GraL})$ was used as the recipient strain. EZ-Tn5' $<$ KAN-2>Tnp Transposome Kit (Epicentre) was used according to the manufacturer's instructions. Transposon insertions were identified by RATE-PCR (Ducey \&Dyer, 2002), with primers listed in Table S2 (at www.actabp.pl).

\section{RESULTS}

\section{In silico prediction of GraL's putative targets and their assessment in vivo}

With the use of the sTarPicker software we were able to identify putative targets of GraL, employing standard parameters suggested at the software webpage (Figs S2 and S3 at www.actabp.pl). A separate search was done for the P1- and P2- promoter initiated GraL transcripts. Nine top rated results for $\mathrm{P} 1$ ( $\lg A, \operatorname{gadB}, n u d E$, panD, rpoS, rs $x C, x a n P, y d c C, y h d V)$ and five top rated results for P2 ( $r: 0 Q, x a n P, y j f P, y j f X, y k g R)$ were chosen for further research. One target $(x a n P)$ was common for both variants, although it is assumed full length GraL would still interact with both types of targets, as the P2 originated transcripts are encompassed in the P1-initiated ones. For the P2 initiated transcript the program mainly predicted targets that would presumably interact with the polyU tail of GraL and which we deem to be nonspecific to GraL. Still, we constructed 13 lac $Z$ fusions of the regions bearing the predicted target sites, with the caveat that the fusion also had to contain the putative gene promoter, any leader region (if applicable) and the sequence corresponding to the first 10 codons of the given gene (Table S2 at www.actabp.pl). The promoter region/target site - lac $Z$ fusions were constructed with the use of the pRS414 plasmid, as described in the Materials and Methods section. The interaction of GraL with putative targets was monitored by measuring $\beta$-galactosidase activity during growth in LB medium, in the presence of a plasmid overproducing GraL (pGraL), its scrambled version ( $\mathrm{pScr}$ ), or a vector control with no insert (pHM1883).

It was anticipated that an interaction of GraL with its target would cause a change in the target fusion's $\beta$-galactosidase activity, corresponding to an increased or decreased ribosome binding site accessibility. Alternatively, the target mRNA stability might have been also affected due to the GraL/mRNA interactions. When present, IPTG was added to $1 \mathrm{mM}$.

Since our previous microarray data indicated that over 100 genes are affected by GraL overexpression in a ppGpp ${ }^{0}$ background, and only very modest effects were observed in the $\mathrm{ppGpp}^{+}$strains (Potrykus et al., 2010), here, we performed initial experiments in the wild type (MG1655 $\Delta$ lacZ, i.e. CF15617) and ppGpp ${ }^{0}$ (MG1655 $\Delta$ lacZ $\Delta$ relA $\Delta s p o T$, i.e. CF15615) strain backgrounds. The $\mathrm{ppGpp}^{0}$ strains are fully devoid of ppGpp (guanosine-3',5'-bisdiphosphate), a crucial mediator of bacterial stringent response (Potrykus \& Cashel, 2008)).

Among all 13 fusions (tested in both strain backgrounds, each carrying pGraL, pScr or the vector control), we found only one where GraL's presence on a multicopy plasmid affected a given target fusion's activity (Fig. 1A, and data not shown). That fusion encompassed nudE region and GraL's effect was evident only in the
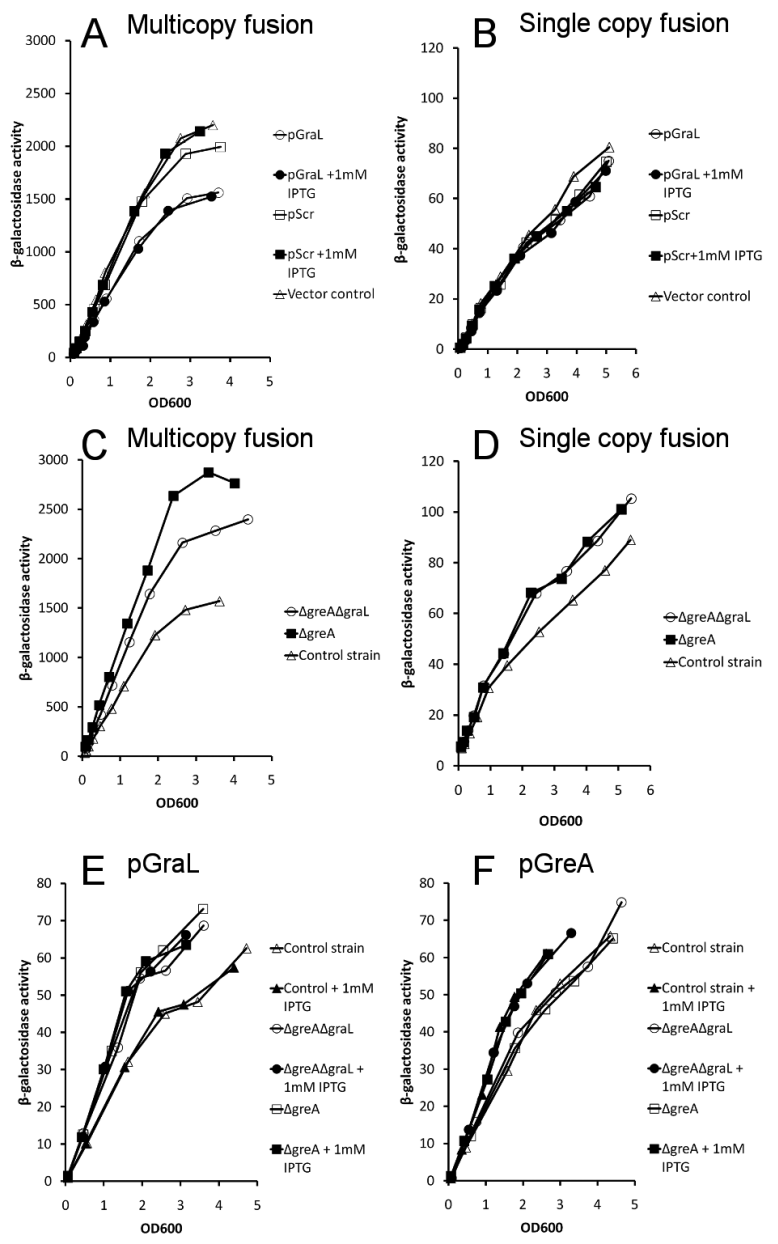

Figure 1. $\beta$-galactosidase activity of the nudE::lacZ fusion in ppGpp $^{0} \Delta$ lacZ strains under different conditions.

Differential plots are depicted, with $\beta$-galactosidase activity plotted on the $y$-axis, and $O D_{600}$ on the $x$-axis. The slope of each curve corresponds to Miller units (specific $\beta$-galactosidase activity, tabulated in Table S3 (at www.actabp.pl for panels A \& B), Table S4 (at www.actabp.pl for panels C \& D) and Table S5 (at www.actabp. pl for panels E \& F)). Cells were inoculated into LB, grown at $30^{\circ} \mathrm{C}$ with shaking, and samples were removed in time. When present, IPTG was added to $1 \mathrm{mM}$ at $\mathrm{OD}_{600} \sim 0.1$. (A) Multicopy nudE::/acZ fusions were employed and activity was monitored under conditions of wt GraL (pGraL, circles) or scrambled GraL (pScr, squares) overproduction. The vector control used was pHM1883 (triangles). Closed symbols indicate addition of IPTG. (B) The same as in (A) but with single copy fusions. (C) Multicopy nudE::lac $Z$ fusions were employed and activity was monitored in $\triangle g r e A$ (squares) and $\triangle$ greA $\triangle G r a L$ (circles) strains. As control, ppGpp $\triangle l a c Z$ strain was used (triangles). (D) The same as in (C) but with single copy fusions. (E) Single copy nudE::lacZ fusions were employed and the effect of GraL's overproduction from the pGraL plasmid was monitored. Control strain - ppGpp $\triangle l a c Z$ (triangles); $\triangle$ greA $\triangle G r a L$ (circles); $\Delta$ greA (squares). Closed symbols indicate addition of IPTG. (F) The same as in (E), but the effect of GreA overproduction from the pGreA plasmid was monitored. 
ppGpp ${ }^{0}$ background, where GraL's overproduction from the plasmid (either in the presence or absence of IPTG) caused a notable, $30 \%$ decrease in the $\beta$-galactosidase activity (decrease in the average Miller units measured over the course of growth, Table S3 at www.actabp.pl and data not shown). The pScr plasmid did not confer such an effect (only about 10\% decrease in the absence of IPTG, and no effect in its presence), indicating the observed inhibition was specific to GraL and did not result simply from titrating out $\mathrm{Hfq}$ or some other regulatory factor, since otherwise overproduction of a random RNA sequence would also cause a drop in activity. Surprisingly, when the nudE::lacZ fusion was then introduced in a single copy on the chromosome, the effect of GraL disappeared (Fig. 1B, Table S3 at www.actabp.pl).

Since the lack of GraL's effect on nudE::lacZ activity, when present in single copy, could be due to the presence of chromosomally encoded GraL, we repeated the above experiment with the multicopy and single copy nudE::lacZ fusions but in the $\triangle$ gre $A \Delta G r a L$ background. A deletion of only GraL would affect the cellular levels of GreA and thus such construct was avoided, as GreA is an important transcription factor. An otherwise ppGpp $^{0}(\mathrm{MG} 1655 \Delta$ lac $\Delta$ relA $\Delta s p o T)$ and $\Delta$ gre $A$ strains were used as control. As can be seen in Fig. 1C and 1D, removal of GraL together with gre $A$ caused an increase in the multicopy nudE::lacZ fusion activity (about 44\% increase in the average Miller units measured over the course of the growth curve, Table S4 at www.actabp. $\mathrm{pl}$. When present in single copy, a much more modest although discernable effect was observed $(12 \%$ increase). Unexpectedly, the removal of only gre $A$ also had an upregulating effect on the nudE::lac $Z$ fusion activity, which was even stronger for the multicopy fusion than the one observed in the $\Delta g r e A \Delta G r a L$ background $(80 \%$ and $13 \%$ increase for the mulicopy and single copy fusions, respectively). No differences were observed in single copy fusion between $\Delta g r e A$ and $\Delta g r e A \Delta G r a L$ strains.

To further test whether GreA itself could affect the $n u d E::$ lac $Z$ fusion activity, we employed the same single copy nudE::lacZ fusion strains as above, but this time either GreA or GraL were overproduced from a multicopy plasmid. Again, a strain with chromosomally encoded gre $A$ and GraL was used as control. As depicted in Fig. $1 \mathrm{~F}$ and Table S5 (at www.actabp.pl), GreA overproduction had only a very modest effect when $1 \mathrm{mM}$ IPTG was added, regardless of whether GraL was present or not (about 13\% increase in all cases). Conversely, when GraL was overproduced from a multicopy plasmid (either in the presence or absence of $1 \mathrm{mM}$ IPTG), the activity of the nudE::lac $Z$ fusion was lower in the presence of chromosomally encoded gre $A$ than in its absence (in the gre $A^{+}$background, the fusion's activity was decreased by about $15.5 \%$ when compared to the $\Delta$ gre $A$ and $\Delta$ gre $A \quad \Delta G r a L$ backgrounds, Fig. $1 \mathrm{E}$ and Table S5 at www.actabp.pl), indicating that to some extent GreA might be involved in the GraL mediated downregulation of the nudE::lac $Z \beta$-galactosidase activity.

\section{In vitro approach to validate GraL-nudE interactions}

In order to validate the above results, we decided to employ electrophoretic mobility shift assays (EMSA) with the use of fluorescently labeled GraL (region spanning from +1 of the gre $A$ P1 promoter to +59$)$ and unlabeled nudE mRNA (see Materials and Methods for details). In the initial experiment, three different nudE mRNA fragments were used - two spanning the predicted GraL binding site ("long" nudE mRNA, spanning
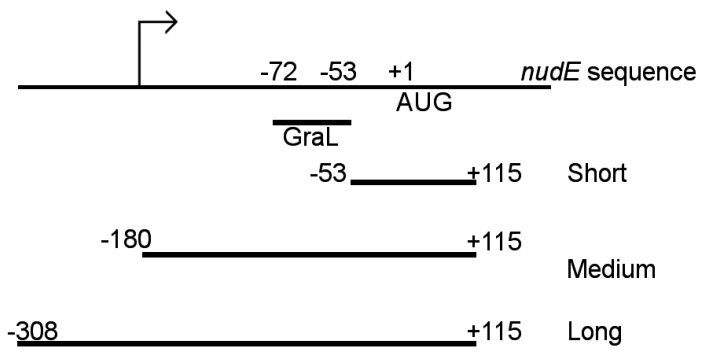

$\operatorname{lacZ}+114+318$

Figure 2. A schematic representation of nudE mRNA fragments used for EMSA assays, with predicted GraL target site indicated; lacZ mRNA (fragment spanning +114 to +318 , based on lacZ present in pUC19) was used as a negative control.

The fragment positions are calculated relative to the first AUG codon of nudE and lacZ mRNAs, respectively. The putative transcription start site is depicted by an arrow, and corresponds to the most upstream putative promoter of nudE (nudEp9); the other four putative promoters are downstream of that region (nudEp6, nudEp8, TSS_3996, nudEp1). The long fragment is spanning the whole region included in the nudE-lacZ fusion.

from -308 to +115 , where +1 is $\mathrm{A}$ in the first $\mathrm{AUG}$ codon of nudE; "medium" nudE mRNA spanning from -180 to +115 ); and the other excluding this site ("short" nudE mRNA, spanning from -53 to +115 ) which we treat as a negative control providing the predicted target site is correct (Fig. 2). As an additional negative control, lacZ mRNA (spanning +114 to +318 ) was used since GraL is not predicted to interact with this mRNA in any way. This would also validate our experiments using the lac $Z$ fusions. GraL was incubated with either of the RNA fragments at a 1:2 ratio, in the absence or presence of Hfq.

As can be seen in Fig. 3, in the absence of Hfq there is an evident decrease in the amount of detectable unbound GraL when either of the nudE fragments is added (about $80 \%$ drop in all cases). On the other hand, no

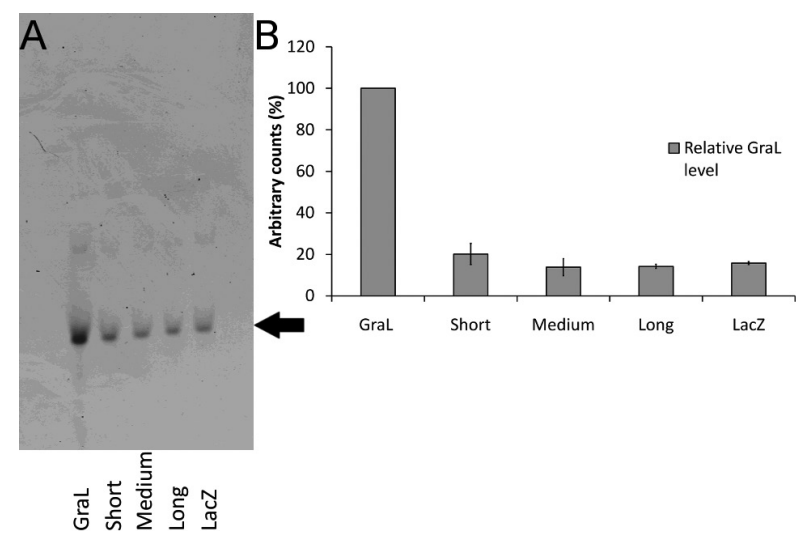

Figure 3. EMSA experiments with GraL and nudE mRNA in the absence of Hfq.

Three nudE mRNAs of different lengths were used ("short" (168 nt), "medium" (295 nt) and "long" (423 nt), as depicted in Fig. 2); lacZ mRNA (204 nt) was used as a negative control. GraL was labeled at the $5^{\prime}$ end with Cy5. The ratio of GraL:nudE mRNA was 1:2. "Control" indicates a control reaction carried out with GraL but without any additional mRNA. (A) A representative EMSA gel; arrow indicates the position of unbound GraL. (B) The amount of unbound GraL is plotted. The data was normalized to the amount of unbound GraL in the absence of any additional mRNA (set as $100 \%)$. The experiment was done in triplicate. Error bars represent S.E.M. 


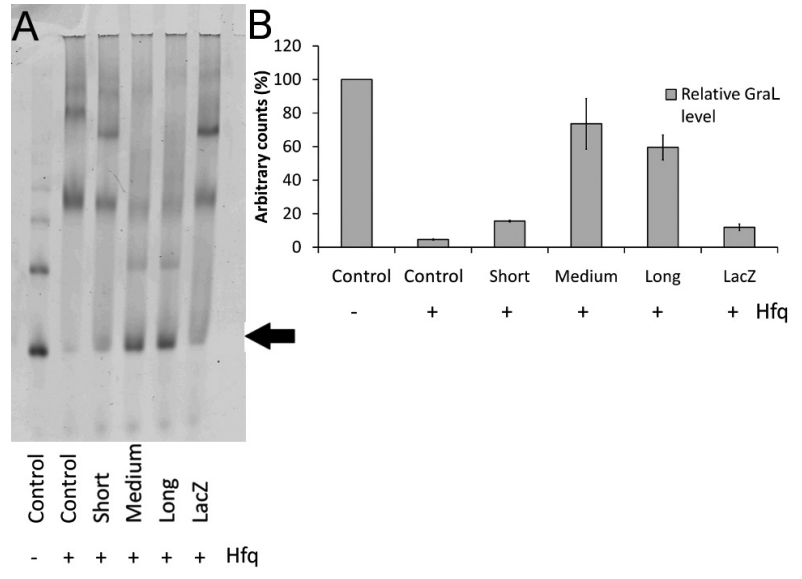

Figure 4. EMSA experiments with GraL and nudE mRNA in the presence of $\mathrm{Hfq}$.

Three nudE mRNAs of different lengths were used ("short", "medium" and "long", as depicted in Fig. 2); lacZ mRNA was used as a negative control. GraL was labeled at the $5^{\prime}$ end with Cy5. The ratio of GraL:nudE mRNA was 1:2. "Control" indicates a control reaction carried out with GraL but without any additional mRNA. (A) A representative EMSA gel; arrow indicates the position of unbound GraL (B) The amount of unbound GraL is plotted. The data was normalized to the amount of unbound GraL in the absence of Hfa and any additional mRNA (set as 100\%).The experiment was done in triplicate. Error bars represent S.E.M.

discernable complexes are detected. Also, a similar effect is observed with the lac $Z$ negative control, which indicates the observed effect is nonspecific. Possibly, due to a high number of such nonspecific GraL-mRNA complexes (spread out throughout each lane), they are below the limit of detection.

Since GraL was recently reported to interact with $\mathrm{Hfq}$ in Salmonella enterica as it was detected among plethora of other sRNAs interacting with this chaperone (Smirnov, 2016), a similar experiment as above was performed in the presence of Hfq (Fig. 4). In the absence of any additional mRNA, about $95 \%$ of GraL is bound by Hfq. Surprisingly, upon adding nudE mRNAs, GraL does not form any additional complexes with slower electrophoretic mobility - instead, GraL seems to be released from the Hfq complexes. This would mean that Hfq has higher affinity towards the nudE fragments used than towards GraL itself and it does not mediate in the GraL-nudE interactions. Indeed, when employing fluorescently labeled nudE mRNA, we found that $\mathrm{Hfq}$ binds to those RNA fragments, and GraL is excluded from those complexes (data not shown). When quantitating unbound GraL, it is evident that the strongest effect is observed for the "medium" and "long" nudE RNAs (about 74\% and 60\% release of GraL from the Hfq complexes, respectively), than for the "short" and lac Z RNAs (only about 16\% and $12 \%$ release, respectively). Thus, this effect seems to be sequence specific, however, it is unclear why GraLnudE complexes of slower electrophoretic mobility again are not being detected. One possibility is that these complexes are too unstable to withstand EMSA conditions.

In another attempt to try to resolve the observed phenomenon, we employed increasing GraL-mRNA ratios, using the "short" and "long" nudE variants (Fig. 5). In the absence of $\mathrm{Hfq}$, as in Fig. 3, we observe a decrease in the unbound GraL amount, which roughly stays at the same level throughout all ratios tested (from 1:2 to 1:16). There are only modest differences between the "short" and "long" nudE RNA. On the other hand, in the Hfq presence, increasing nudE RNA concentrations

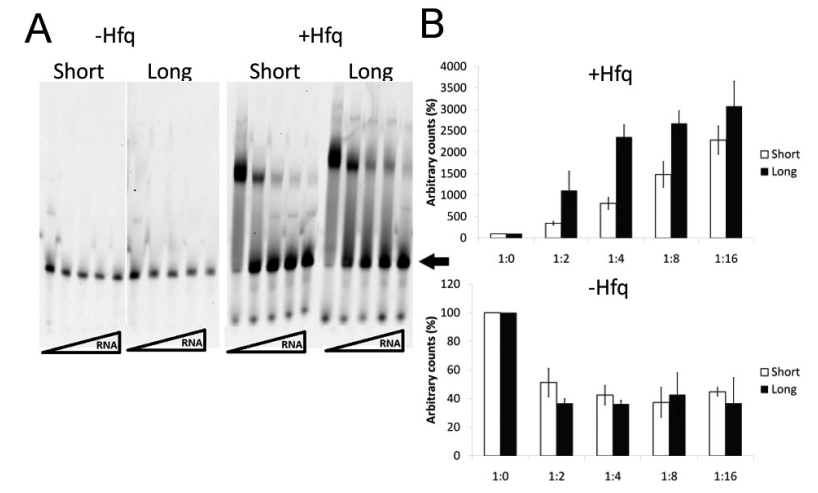

Figure 5. Titration of two nudE mRNA variants in the presence or absence of Hfq.

GraL was labeled at the 5' end with Cy5. "Short" and "long" nudE variants were used (see Fig. 2). The ratios of GraL:nudE were $1: 2,1: 4,1: 8$ and 1:16. (A) Representative EMSA gels; arrow indicates the position of unbound GraL (B) The amount of unbound GraL obtained in the absence or presence of $\mathrm{Hfq}$ is plotted. The data was normalized to the amount of unbound GraL at the 1:0 GraL:nudE ratio (set as $100 \%$ ). The experiment was done in duplicate. Error bars represent S.E.M.

seem to release more GraL from the GraL-Hfq complexes (Fig. 5). As in Fig. 4, this effect seems to be sequence specific, since "long" nudE RNA is far better at competing out Hfq than the "short" version (especially when present at the 1:2, 1:4 and 1:8 ratios). However, as before, the presumable GraL-nudE complexes are not discernable.

\section{Search for genes that are synthetic lethal with GraL}

Screens for synthetic lethal genes help to define networks of interactions between genes whose products might be involved in the same processes such that one can compensate for the other under certain circumstances. Such screens are especially important for genes whose deletion (or inactivation) on their own does not confer an apparent phenotype, or the phenotype is minor, as is the case for GraL.

In our screen, we had employed a low copy number, unstable plasmid (pRC7 (Bernhardt \& de Boer, 2005)) which is easily lost by the cells in the absence of antibiotic pressure (i.e. ampicillin, as the plasmid confers ampicillin resistance). In addition, the pRC7 plasmid also carries lac $Z Y A$, so that its presence can be monitored in E. coli cells lacking lacZ on the chromosome by simply plating cells on a medium supplemented with X-gal. We had cloned GraL into this plasmid under $\mathrm{p}_{\text {lac }}$ promoter. GraL overproduction from the resulting pRCGraL plasmid was confirmed by Northern blot analysis (data not shown).

In our initial experiments we wanted to test whether pRC-GraL would be indeed easily lost by bacterial cells in our experimental setup. The strains we employed, besides having deleted lac $Z$ on the chromosome, were also either $\Delta g r e A$ (control) or $\Delta$ gre $A \Delta G r a L$. In addition, we also tested the effect of overproducing GreA from a plasmid (pGreA), as originally we assumed we would perform our screen in the $\Delta G r a L$ gre $A^{+}$background. $\mathrm{ppGpp}^{+}$and $\mathrm{ppGpp}^{0}$ strains were employed, bringing the number of the tested combinations to 16 strains, including controls (Table S1 at www.acyabp.pl).

Appropriate strains were inoculated into liquid LB medium without ampicillin to obtain $\mathrm{OD}_{600} \sim 0.05$. Growth was continued with aeration for $3 \mathrm{hrs}$, and samples were 
withdrawn every $30 \mathrm{~min}$. The cells were then plated on $\mathrm{LB}$ with $\mathrm{X}$-gal, incubated at $30^{\circ} \mathrm{C}$ for $48 \mathrm{hrs}$ and the color of colonies was scored. Completely white colonies indicated that the initial cells plated on the medium had already lost the plasmid; completely blue colonies were those where each cell had retained the plasmid throughout growth on plate; sectioned colonies (partly white, partly blue), corresponded to an instance when some of the cells had lost the plasmid while the colony was growing on the plate.

As depicted in Figs S4 and S5 (at www.actabp.pl), the strains that lost the pRC-GraL plasmid the most easily were $\mathrm{ppGpp}^{0} \Delta$ gre $A \quad \Delta G r a L$ and $\mathrm{ppGpp}^{0} \Delta$ gre $A$ $(81 \%$ and $78 \%$ of colonies were fully or partly devoid of pRC-GraL after $3 \mathrm{hr}$ cultivation in LB without ampicillin, respectively). Surprisingly, overproduction of GreA from a plasmid seemed to stabilize pRC-GraL in these strains, as more of the completely blue colonies formed in its presence $(19 \%$ and $21 \%$ without pGreA vs $65 \%$ and $63 \%$ with pGreA, respectively), and thus pGreA was omitted in further studies.

In order to search for genes that are synthetic lethal with GraL, we performed random transposon insertion mutagenesis with the Tn5::kan transposon and the $\mathrm{ppGpp}^{0} \Delta$ greA $\Delta \mathrm{GraL} / \mathrm{pRC}-\mathrm{GraL}$ strain (see Materials and Methods section). The mutant library was first plated on LB containing kanamycin and ampicillin (to select for clones carrying both, Tn5::kan and pRC-GraL), and was estimated to have 18,440 individual clones. Thus obtained colonies were collected, pooled and then plated on LB without any antibiotic, but containing X-gal. The assumption is that if the transposon happened to disrupt a gene whose lack of function renders GraL to be essential, a completely blue colony would arise, as the plasmid would have to be maintained in all cells of a given colony.

The screen was carried out twice, in the first instance screening about 10000 colonies, and screening about 20000 colonies in the second one. After initial plating on X-gal plates, 80 and 100 insertion mutant clones qualified for further analysis, in the first and second screen, respectively. Upon subsequent restreaking, done twice for each selected clone, 20 and 30 mutant strains maintained pRC-GraL in all cells of all of their colonies in streaks on plates, in the first and second screen, respectively. The genes where the transposon was inserted were then identified by RATE-PCR and subsequent sequencing. This approach was successful for 18 and 29 clones, in the first and second screen, respectively. Altogether, we found that 40 genes seem to be synthetic lethal with GraL. In four instances the same gene was found to be disrupted in both screens $(e c p C, f e o A, x a n P$, $y i a F)$, and in three instances the same gene was identified twice in the same screen (feo $A$, narZ, $y h f T$ ). The results are presented in Table 1.

\section{DISCUSSION}

In the work presented here, we have undertaken to identify the regulatory role of GraL in trans to substantiate its biological significance that has been previously demonstrated with microarray and fitness assays (Potrykus et al., 2010). Here, we identified nudE as a potential target of GraL in vivo, as we show that GraL overproduction decreases the nudE::lacZ fusion activity, when the fusion is present in multicopy. For the single copy fusion, this effect is observed only in cells devoid of GraL on the chromosome (in this case, nudE::lacZ ac- tivation is observed). This could indicate the amount of GraL supplied from the chromosomally encoded sRNA is enough to exert regulation. Surprisingly, GreA seems to play a part in the nudE regulation as well, since deleting only gre $A$ also upregulates $\beta$-galactosidase activity of the nudE::lacZ fusion. On the other hand, GreA overexpression also has an upregulatory effect. This could indicate the existence of a feed-back regulatory loop involving GraL and GreA. More research is needed to understand this possible phenomenon. The fact that GraL is encoded in the gre $A$ leader region and regulates the amount of gre $A$ mRNA that is transcribed, further complicates this matter.

In an effort to confirm our in vivo observations, we performed a series of in vitro experiments employing electrophoretic mobility shift assays (EMSA). We found that Hfq binds to GraL, which is in line with a recently published report on Salmonella enteritica sRNA that bind Hfq or ProQ (a newly discovered sRNA chaperone protein) (Smirnov et al., 2016). There, by employing gradient profiling of protein-bound sRNA, GraL was identified among sRNA that are bound by Hfq. Since GraL is highly conserved in the enteric bacteria (Potrykus et al., 2010), it could be expected that binding of Hfq to GraL would also hold true for E. coli. Still, in our EMSA experiments we found that Hfq which was pre-bound to GraL could be easily out-competed by nudE mRNA, although we did not detect any recognizable GraL-nudE complexes, while at the same time the added mRNA did shift with Hfq itself (this complex did not include GraL; data not shown). This is also in line with evidence that sRNAs may actively compete with each other for binding to Hfq (Fender et al., 2010; Moon \& Gottesman, 2011). Overall, we take the results obtained here by us to mean that although GraL is bound by Hfq, this chaperone does not mediate the GraL-nudE interactions.

The nudE gene, identified in our in silico search and in vivo tests as a possible target for GraL, encodes an adenosine nucleotide hydrolase, which is a nudix enzyme whose substrates include NADH, ADP-D-ribose, and 5',5'"-diadenosine triphosphate (Ap3A) (O'Handley et al., 1998). A decrease in nudE expression could possibly lead to an imbalance in the $\mathrm{NADH} / \mathrm{NAD}^{+}$ratios and in effect have broad cellular implications, as NAD serves as a cofactor in over 300 red-ox reactions (Foster et al., 1990). On the other hand, it could be also imagined that decreased nudE expression would yield increased levels of ADP-D-ribose in the cell, which in turn could lead to increased non-enzymatic protein glycation and thus protein inactivation and/or enhanced degradation (O'Handley et al., 1998). In that second instance, however, a disadvantage to cells would have been expected, while cells overexpressing GraL were characterized by better fitness than wild type cells (Potrykus et al., 2010).

Our search for genes that are synthetic lethal with GraL, i.e. in the absence of which GraL becomes essential for the cell, returned 40 genes that could be roughly divided into 5 major categories: metabolism (carbohydrate, amino acid, energy and lipid metabolism - 14 genes), environmental information processing (signal transduction and membrane transport - 6 genes), transporters ( 7 genes), transcription factors (5 genes) and enzymes (12 genes; among them, transferases seem to be the most abundant -6 genes). Evidently, no clear correlation between the functions of these genes is found. Instead, GraL indeed seems to affect many different processes, as would be expected if the NADH/ $\mathrm{NAD}^{+}$ratios were altered. It is possible that in the absence of GraL, NADH levels would decrease in the cell 
Table 1. Genes identified as synthetic lethal with GraL.

"Descriptions are provided according to the KEGG Pathway and KEGG BRITE annotation (http://www.genome.jp/kegg/); "Several genes are annotated in multiple ways, thus they are listed more than once; ${ }^{\circledR}$ genes found twice in the same screen; ${ }^{*}$ genes found in both screens; genes in parenthesis - not classified in KEGG, but listed here according to EcoGene (www.ecogene.org (Zhou \& Rudd, 2013))

\begin{tabular}{|c|c|}
\hline Description" & Gene" \\
\hline \multicolumn{2}{|l|}{ Environmental Information Processing } \\
\hline Signal Transduction & $c p x R, g \ln A, n a r Z^{\S}$ \\
\hline Membrane transport & fecC, tauA, yhbG \\
\hline \multicolumn{2}{|l|}{ Enzymes } \\
\hline Hydrolases & $y h b G$ \\
\hline Ligases & $g \ln A$ \\
\hline Lyases & $f a b A$ \\
\hline Oxidoreductases & adhE, bisC, narZs \\
\hline Transferases & cysD, maa, pflD, tdcE, waaP, yahl \\
\hline Lipopolysaccharide biosynthesis proteins & waaP \\
\hline \multicolumn{2}{|l|}{ Metabolism } \\
\hline \multicolumn{2}{|l|}{ Amino acid metabolism } \\
\hline Alanine, aspartate and glutamate metabolism & $g \ln A$ \\
\hline Arginine biosynthesis & $g \ln A$, yahl \\
\hline Metabolism of other amino acids & $c y s D$ \\
\hline Tyrosine metabolism & adhE \\
\hline \multicolumn{2}{|l|}{ Carbohydrate metabolism } \\
\hline Galactose metabolism & gatZ \\
\hline Glycan biosynthesis and metabolism & waaP \\
\hline Glycolysis/gluconeogenesis & adhE \\
\hline Glyoxylate and dicarboxylate metabolism & $g \ln A$ \\
\hline Pyruvate metabolism & adhE, eutD, pflD, tdcE \\
\hline \multicolumn{2}{|l|}{ Energy metabolism } \\
\hline Nitrogen metabolism & $g \ln A, n a r Z^{\S}$, yahl \\
\hline Nucleotide metabolism & cysD, yahl \\
\hline Sulfur metabolism & cys $D, \operatorname{tau} A$ \\
\hline Lipid metabolism & $a d h E, f a b A$ \\
\hline Metabolism of cofactors and vitamins & bisC \\
\hline Transcription factors & cadC, frlR, (perR), tdcA, (yahB) \\
\hline \multicolumn{2}{|l|}{ Transporters } \\
\hline$A B C$ transporters & fecC, tauA, yhbG \\
\hline Other transporters & bisC, feo ${ }^{\S *}, g l c A, x a n P^{*}$ \\
\hline Secretion system & $e c p C^{*}$ \\
\hline Other/ unclassified & $\begin{array}{l}r b b A, r c l C, r n I A, s s I E, \text { yaiO, ydeP, ydfE, ygaU, ygbT, yhfTs, yiaF*, yjbG, } \\
\text { yjcH, ypjA }\end{array}$ \\
\hline
\end{tabular}

which would be lethal to cells carrying mutations in the genes that we identified in our screen. Providing GraL on a plasmid would decrease nudE expression, returning $\mathrm{NADH} / \mathrm{NAD}^{+}$ratios to a relatively normal level and thus rescuing cell growth. On the other hand, it should be kept in mind that disruption of a given gene by transposon insertion might have polar effects on expression of the neighboring genes, and in fact disturbance in their expression and not of the genes identified here might be key to rendering GraL as essential for the cell. Future work should focus on testing non-polar deletions of the genes identified here, as well as testing whether gre $A$ de- letion is required for the observed synthetic lethality to occur.

Recently, a report was published where a RIL-seq method was used to identify E. coli sRNA-mRNA target pairs that require $\mathrm{Hfq}$ to mediate their interaction (Melamed et al., 2016). This method relies on immunoprecipitation of $\mathrm{Hfq}$ bound with both RNA molecules. The experiments were performed under three different conditions: logarithmic phase of growth, stationary phase and iron limitation. Thousands of sRNA-mRNA interactions were scored, and among them one seems to involve GraL. That interaction was detected under stationary phase conditions for +34 to +54 nts of GraL and 
an intergenic region between $g c v H$ and $g c v P$ (Melamed et al., 2016). The gcvP gene encodes glycine dehydrogenase, and it could be imagined that GraL's pairing with the $g c v H-g c v \mathrm{P}$ intergenic region mRNA might affect $g c v \mathrm{P}$ expression by affecting the mRNA stability. Still, although very intriguing, this interaction involves the so-called $\mathrm{BOXC}$, i.e. a GC rich intergenic repeat, likely involved in genome instability (Holder et al., 2015). When we used IntaRNA software (Wright et al., 2014) to predict GraLmRNA interactions with this region, no possible interactions were found. Therefore, we take the result obtained in the (Melamed et al., 2016) search as an artifact, although it cannot be excluded that GraL pairs with its targets in a non-canonical way.

One intriguing possibility is that GraL acts as an aptamer, directly binding to RNA polymerase and affecting its activity, as has been recently shown for a set of small RNAs (Sedlyarova et al., 2017). There, the inhibitory aptamers were cis-encoded, however, it cannot be excluded that aptamers acting in trans also exist. This could explain GraL's pleotropic effects and the fact that this sRNA's deletion is synthetic lethal with genes whose products participate in many different processes.

We had also performed additional in silico searches with more recently released software, such as CopraRNA (Wright et al., 2014) and TargetRNA2 (Kery et al., 2014). However, we did not obtain any satisfactory results, i.e. the targets in each search were multiple and very different, often relying on interactions with GraL's polyU tail, which we deem to be nonspecific; we did not find any significant putative GraL targets that would be in common for those programs, other than what we initially obtained by using the sTarPicker software (data not shown). The CopraRNA results were especially disappointing since this program relies on search made within related organisms, assuming that if an sRNA is conserved, the targets should be also conserved, and thus the obtained results could be classified as highly probable. We found significant differences depending on how many strains were included in the search (it is required to include at least three and at most five different species), and what is more, all of the results but one returned with a very high false discovery rate index $(0.95$; the following strains were compared: E. coli as the reference strain and then - Shigella dysenteriae, Shigella boydi, Salmonella enterica, Yersinia pestis; data not shown). The one result that could be of any significance was rpos, which we had already tested in our in vivo assays and which turned out not to be a target for GraL (above and data not shown).

One might say that since GraL is highly conserved among enteric bacteria, it cannot be excluded that GraL's role in cis is the one that is highly conserved (i.e. gre $A$ expression regulation) rather than its role in trans. Still, as has been demonstrated previously, GraL affects expression of many E. coli genes in trans, and as we show here, its deletion is synthetic lethal with a number of genes participating in different processes, indicating GraL itself plays an important role in the cell. All of the above seem to indicate that GraL acts in a non-canonical way and further research is necessary to elucidate the mechanism of its action.

\section{Aknowledgements}

We thank Dr. Grzegorz M. Cech for the generous gift of purified Hfq protein. We gratefully acknowledge Dr. Rajendran Harinarayanan for suggesting using RATEPCR to identify the random transposon insertion library mutants. We also thank Dr. Bożena Bruhn-Olszewska, Michał Sobala (M.Sc), Dr. Mike Cashel and Dr. Llorenc Fernandez Coll for helpful discussions.

\section{Acknowledgements of Financial Support}

This work was funded by the National Science Centre (Poland), grant no. UMO-2013/09/B/NZ1/01066 to K.P.

\section{REFERENCES}

Aberg A, Shingler V, Balsalobre C (2008) Regulation of the fimB promoter: a case of differential regulation by ppGpp and DksA in vivo. Mol Microbiol 67: 1223-1241. http://doi.org/10.1111/j.13652958.2006.05191.x

Beisel CL, Updegrove TB, Janson BJ, Storz G (2012) Multiple factors dictate target selection by Hfq-binding small RNAs. EMBO J 31: 1961-1974. http://doi.org/10.1038/emboj.2012.52

Bernhardt TG, de Boer PA (2005) SlmA, a nucleoid-associated, FtsZ binding protein required for blocking septal ring assembly over chromosomes in E. coli. Mol Cell 18: 555-564. https://doi. org/10.1016/j.molcel.2005.04.012

Borukhov S, Lee J, Laptenko O (2005) Bacterial transcription elongation factors: new insights into molecular mechanism of action. Mol Microbiol 55: 1315-1324. https://doi.org/10.1111/j.13652958.2004.04481.x

Churchward G, Belin D, Nagamine, Y (1984) A pSC101-derived plasmid which shows no sequence homology to other commonly used cloning vectors. Gene 31: 165-171. https://doi.org/10.1016/03781119(84)90207-5

de Boer PAJ, Crossley RE, Rothfield, LI (1989) A division inhibitor and a topological specificity factor coded for by the minicell locus determine proper placement of the division septum in E. coli. Cell 56: 641-649. https://doi.org/10.1016/0092-8674(89)90586-2

Ducey TF, Dyer DW (2002) Rapid identification of EZ::TNTM transposon insertion sites in the genome of Neisseria gonorrboeae. EPICENTRE Forum 9: 6-7.

Faner MA, Feig AL (2013) Identifying and characterizing Hfq--RNA interactions. Methods pii: S1046-2023(13)00140-0. https://doi. org/10.1016/j.ymeth.2013.04.023

Fender A, Elf J, Hampel K, Zimmermann B, Wagner EGH (2010) RNAs actively cycle on the Sm-like protein Hfq. Genes Dev 24: 2621-2626. https://doi.org/10.1101/gad.591310

Foster JW, Park YK, Penfound T, Fenger T, Spector MP (1990) Regulation of NAD metabolism in Salmonella typhimurium: molecular sequence analysis of the bifunctional nadR regulator and the nadApnuC operon. J Bacteriol 172: 4187-4196

Gottesman S (2004) The small RNA regulators of Escherichia coli: roles and mechanisms. Annu Rev Microbiol 58: 303-328. https://doi. org/10.1146/annurev.micro.58.030603.123841

Gottesman S, Storz G (2011) Bacterial small RNA regulators: versatile roles and rapidly evolving variations. Cold Spring Harb Perspect Biol 3: a003798. https://doi.org/10.1101/cshperspect.a003798

Guillier M, Gottesman S (2006) Remodelling of the Escherichia coli outer membrane by two small regulatory RNAs. Mol Microbiol 59: 231-247. https://doi.org/10.1111/j.1365-2958.2005.04929.x

Hoe CH, Raabe CA, Rozhdestvensky TS, Tang TH (2013) Bacterial sRNAs: Regulation in stress. Int J Med Microbiol. pii: S14384221(13)00055-6. https://doi.org/10.1016/j.ijmm.2013.04.002

Holder IT, Wagner S, Xiong P, Sinn M, Frickey T, Meyer A, Hartig JS (2015) Intrastrand triplex DNA repeats in bacteria: a source of genomic instability. Nucleic Acids Res 43: 10126-10142. https://doi. org/10.1093/nar/gkv1017

Huang HY, Chang HY, Chou CH, Tseng CP, Ho SY, Yang CD, Ju YW, Huang HD (2009) sRNAMap: genomic maps for small noncoding RNAs, their regulators and their targets in microbial genomes. Nucleic Acids Res 37: D150-D154. https://doi.org/10.1093/nar/ gkn852

Kawano M, Oshima T, Kasai H, Mori H (2002) Molecular characterization of long direct repeat (LDR) sequences expressing a stable mRNA encoding for a 35-amino-acid cell-killing peptide and a cis-encoded small antisense RNA in Escherichia coli. Mol Microbiol 45: 333-349. https://doi.org/10.1046/j.1365-2958.2002.03042.x

Kery MB, Feldman M, Livny J, Tjaden B (2014) TargetRNA2: identifying targets of small regulatory RNAs in bacteria. Nucleic Acids Res 4: W124-W129. https://doi.org/10.1093/nar/gku317

Kortmann J, Narberhaus F. (2012) Bacterial RNA thermometers: molecular zippers and switches. Nat Rev Microbiol 10: 255-265. https:// doi.org/10.1038/nrmicro2730

Laptenko O, Lee J, Lomakin I, Borukhov S (2003). Transcript cleavage factors GreA and GreB act as a transient catalytic compo- 
nents of RNA polymerase. EMBO J 22: 6322-6334. https://doi. org/10.1093/emboj/cdg610

Lease RA, Cusick ME, Belfort M (1998) Riboregulation in Escherichia coli: DsrA RNA acts by RNA:RNA interactions at multiple loci. Proc Natl Acad Sci U S A 95: 12456-12461. https://doi.org/10.1073/ pnas.95.21.12456

Majdalani N, Vanderpool CK, Gottesman S (2005) Bacterial small RNA regulators. Crit Rev Biochem Mol Biol 40: 93-113. https://doi. org/10.1080/10409230590918702

Massé E, Salvail H, Desnoyers G, Arguin M (2007) Small RNAs controlling iron metabolism. Curr Opin Microbiol 10: 140-145. https:// doi.org/10.1016/j.mib.2007.03.013

Masse E, Vanderpool CK, Gottesman S (2005) Effect of RyhB small RNA on global iron use in Escherichia coli. J Bacteriol 187: 6962-6971. https://doi.org/10.1128/JB.187.20.6962-6971.2005

McCullen CA, Benhammou JN, Majdalani N, Gottesman S (2010) Mechanism of positive regulation by DsrA and RprA small noncoding RNAs: pairing increases translation and protects rpos mRNA from degradation. J Bacteriol 192: 5559-5571. https://doi. org/10.1128/JB.00464-10

Melamed S, Peer A, Faigenbaum-Romm R, Gatt YE, Reiss N, Bar A, Altuvia Y, Argaman L, Margalit H (2016) Global mapping of small RNA-target interactions in bacteria. Mol Cell 63: 884-897. https:// doi.org/10.1016/j.molcel.2016.07.026

Miller JH (1972) Experiments in molecular genetics. Cold Spring Harbor Laboratory Press, Cold Spring Harbor, NY.

Morita T, Maki K, Aiba H (2012) Detection of sRNA-mRNA interactions by electrophoretic mobility shift assay. Methods Mol Biol 905: 235-244. https://doi.org/10.1007/978-1-61779-949-5_15

Moon K, Gottesman S (2009) A PhoQ/P-regulated small RNA regulates sensitivity of Escherichia coli to antimicrobial peptides. Mol Microbiol 74: 1314-1330. https://doi.org/10.1111/j.13652958.2009.06944.x.

Moon K, Gottesman S (2011) Competition among Hfq-binding small RNAs in Escherichia coli. Mol Microbiol 82: 1545-1562. https://doi. org/10.1111/j.1365-2958.2011.07907.x

O’Handley SF, Frick DN, Dunn CA, Bessman MJ (1998) Orf186 represents a new member of the Nudix hydrolases, active on adenosine(5')triphospho(5')adenosine, ADP-ribose, and NADH. J Biol Chem 273: 3192-3197

Pedersen K, Gerdes K (1999) Multiple bok genes on the chromosome of Escherichia coli. Mol Microbiol 32: 1090-1102. https://doi. org/10.1046/j.1365-2958.1999.01431.x

Potrykus K, Cashel M (2008) (p)ppGpp: still magical? Annu Rev Microbiol 62: 35-51. https://doi.org/10.1146/annurev.micro.62.081307.162903

Potrykus K, Murphy H, Chen X, Epstein JA, Cashel M (2010) Imprecise transcription termination within Escherichia coli gre $A$ leader gives rise to an array of short transcripts, GraL. Nucleic Acids Res. 38: 1636-1651. https://doi.org/10.1093/nar/gkp1150

Potrykus K, Vinella D, Murphy H, Szalewska-Palasz A, D’Ari R, Cashel M (2006) Antagonistic regulation of Escherichia coli ribosomal RNA rmB P1 promoter activity by GreA and DksA. I Biol Chem 281: 15238-15248. https://doi.org/10.1074/jbc.M601531200

Powell BS, Rivas MP, Court DL, Nakamura Y, Turnbough CL Jr. (1994) Rapid confirmation of single copy lambda prophage integration by PCR. Nucleic Acids Res 22: 5765-5766.

Romby P, Vandenesch F, Wagner EG (2006) The role of RNAs in the regulation of virulence-gene expression. Curr Opin Microbiol 9: 229236. https://doi.org/10.1016/j.mib.2006.02.005
Sedlyarova N, Rescheneder P, Magan A, Popitsch N, Rziha N, Bilusic I, Epshtein V, Zimmermann B, Lybecker M, Sedlyarov V, Schroeder R, Nudler E (2017) Natural RNA polymerase aptamers regulate transcription in E. coli. Mol Cell 67: 3043. https://doi.org/10.1016/j. molcel.2017.05.025

Sharan SK, Thomason LC, Kuznetsov SG, Court DL (2009) Recombineering: a homologous recombination-based method of genetic engineering. Nat Protoc 4: 206223. https://doi.org/10.1038/ nprot.2008.227

Simons RW, Houman F, Kleckner N (1987) Improved single and multicopy lac-based cloning vectors for protein and operon fusions. Gene 53: 85-96. https://doi.org/10.1016/0378-1119(87)90095-3

Sledjeski D, Gottesman S (1995) A small RNA acts as an antisilencer of the H-NS-silenced rcs A gene of Escherichia coli. Proc Natl Acad Sci US A 92: 2003-2007

Smirnov A, Forstner KU, Holmqvist E, Otto A, Gunster R, Becher D, Reinhardt R, Vogel J (2016) Grad-seq guides the discovery of ProQ as a major small RNA-binding protein. Proc Natl Acad Sci U S A 113: 11591-11596. https://doi.org/10.1073/pnas.1609981113

Storz G, Vogel J, Wassarman KM (2011) Regulation by Small RNAs in Bacteria: Expanding Frontiers. Mol Cell 43: 880-891. https://doi. org/10.1016/j.molcel.2011.08.022

Studier FW (1991) Use of bacteriophage T7 lysozyme to improve an inducible T7 expression system. J Mol Biol 219: 37-44. https://doi. org/10.1016/0022-2836(91)90855-Z

Tjaden B, Goodwin SS, Opdyke JA, Guillier M, Fu DX, Gottesman S, Storz G (2006) Target prediction for small, noncoding RNAs in bacteria. Nucleic Acids Res 34: 2791-2802. https://doi.org/10.1093/ nar/gkl356

Toledo-Arana A, Repoila F, Cossart P (2007) Small noncoding RNAs controlling pathogenesis. Curr Opin Microbiol 10: 182-188. https:// doi.org/10.1016/j.mib.2007.03.004

Tu K, Bassler BL (2007) Multiple small RNAs act additively to integrate sensory information and control quorum sensing in Vibrio harveyi. Genes Dev 21: 221-233. https://doi.org/10.1101/gad.1502407

Valentin-Hansen P, Johansen J, Rasmussen AA (2007) Small RNAs controlling outer membrane porins. Curr Opin Microbiol 10: 152-155. https://doi.org/10.1016/j.mib.2007.03.001

Vecerek B, Moll I, Bläsi U (2007) Control of Fur synthesis by the non-coding RNA RyhB and iron-responsive decoding. EMBO J 26: 965-975. https://doi.org/10.1038/sj.emboj.7601553

Vinella D, Potrykus K, Murphy H, Cashel M (2012) Effects on growth by changes of the balance between GreA, GreB, and DksA suggest mutual competition and functional redundancy in Escherichia coli. J Bacteriol 194: 261-273. https://doi.org/10.1128/JB.06238-11

Wright PR, Georg J, Mann M, Sorescu DA, Richter AS, Lott S, Kleinkauf R, Hess WR, Backofen R (2014) CopraRNA and IntaRNA: predicting small RNA targets, networks and interaction domains. Nucleic Acids Res 42: W119-W123. https://doi.org/10.1093/ nar/gku359

Ying X, Cao Y, Wu J, Liu Q, Cha L, Li W (2011) sTarPicker: a method for efficient prediction of bacterial sRNA targets based on a two-step model for hybridization. PLoS One 6: e22705. https://doi. org/10.1371/journal.pone.0022705

Zhou J, Rudd K (2013) EcoGene 3.0. Nucleic Acids Res 41: D613-D624. https://doi.org/10.1093/nar/gks1235 